BULL. AUSTRAL. MATH. SOC.

MOS $8035,{ }^{*} 8030,3579,3511$

VOL. I (1969), 144

\title{
Equations of heat conduction with slow combustion
}

\author{
Graeme C. Wake
}

A study is made of the equations of heat conduction with slow combustion. A mathematical model is established from an interpretation of the physical model, with a few simplifying assumptions. This gives rise to a coupled pair of partial differential equations which are the direct concern of this thesis, the dependent variables being the temperature and reactant concentration as functions of position and time.

The model is shown to possess a unique solution for which some properties, such as Lipschitz conditions, etc., are established. An investigation into the use of a comparison theorem is given, in which it is shown that no direct comparison theorem is possible for this and related systems. However, it is also shown that it is possible to obtain upper and lower estimates by appealing to the physical model.

A discussion of the boundary layer is given and this is followed by a detailed discussion of stability. The latter has been one of the main concerns of earlier authors on this system. Their use of a space-averaging process to establish a criterion for stability is also discussed.

Probably one of the most interesting features of this system is the subclass of problems for which the reactant is exhausted in a finite time. These have been named the "cut-off" problems and they can be likened to the free boundary problems in fluid dynamics. A discussion of the cut-off problem is given with particular examples chosen to illustrate the main features.

Received 24 March 1969. Thesis submitted to the Victoria University of Wellington, January 1967. Degree approved, October 1967. Supervisors: Professor A.G. Mackie, Mr A. McNabb. 